\title{
GATA-dependent transcriptional and epigenetic control of cardiac lineage specification and differentiation
}

\author{
Sonia Stefanovic ${ }^{1} \cdot$ Vincent M. Christoffels $^{1}$
}

Received: 20 October 2014/Revised: 15 June 2015/ Accepted: 17 June 2015/Published online: 1 July 2015

(C) The Author(s) 2015. This article is published with open access at Springerlink.com

\begin{abstract}
Heart progenitor cells differentiate into various cell types including pacemaker and working cardiomyocytes. Cell-type specific gene expression is achieved by combinatorial interactions between tissue-specific transcription factors (TFs), co-factors, and chromatin remodelers and DNA binding elements in regulatory regions. Dysfunction of these transcriptional networks may result in congenital heart defects. Functional analysis of the regulatory DNA sequences has contributed substantially to the identification of the transcriptional network components and combinatorial interactions regulating the tissuespecific gene programs. GATA TFs have been identified as central players in these networks. In particular, GATA binding elements have emerged as a platform to recruit broadly active histone modification enzymes and cell-typespecific co-factors to drive cell-type-specific gene programs. Here, we discuss the role of GATA factors in cell fate decisions and differentiation in the developing heart.
\end{abstract}

Keywords Heart development - Cell fate decisions . Histone-modifying enzymes - GATA transcription factor . Chambers · Conduction system

\section{Abbreviations \\ ANF Atrial natriuretic factor \\ AV Atrioventricular}

Sonia Stefanovic

s.stefanovic@amc.uva.nl

$\triangle$ Vincent M. Christoffels

v.m.christoffels@amc.uva.nl

1 Department of Anatomy, Embryology and Physiology, Academic Medical Center, University of Amsterdam, Meibergdreef 15, 1105 AZ Amsterdam, The Netherlands

$\begin{array}{ll}\text { BMP } & \text { Bone morphogenetic peptide } \\ \text { BNF } & \text { Brain natriuretic factor } \\ \text { E } & \text { Embryonic day } \\ \text { HAT } & \text { Histone acetyl transferase } \\ \text { HDAC } & \text { Histone deacetylase } \\ \text { TBE } & \text { T-box element } \\ \text { TF } & \text { Transcription factor }\end{array}$

\section{Introduction}

Congenital heart defects affect nearly one percent of live births and are found in up to one-tenth of spontaneously aborted fetuses $[1,2]$. The development of the heart is controlled by evolutionary conserved transcriptional networks. Genetic studies have identified mutations in genes encoding transcription factors as well as proteins organizing the chromatin structure that are responsible for congenital heart defects [3-5]. Defining the transcriptional networks underlying normal heart development is a prerequisite for understanding the molecular basis of congenital heart malformation. The specification of multipotent heart progenitor cells and their differentiation into different cell lineages is under tight spatial and temporal transcriptional control. Regulatory DNA elements (promoters, enhancers, repressors, and insulators/boundary elements) can interact with and respond to activating or repressing transcriptional complexes (transcription factors, activators, coactivators, repressors, and corepressors) to mediate the temporal and spatial control of transcription. Hence, functional analysis of DNA elements conferring cell-type-specific gene expression is a good starting point to identify the molecular mechanisms that underlie these patterns of gene expression. Dissection and analysis of regulatory DNA elements have led to the identification of 
many key regulators of the cardiac gene regulatory programs including the zinc finger TF Gata4. Mutations in both GATA4 and its family member GATA6 in patients have been associated with arrhythmias and defects in atrial, ventricular, and AV septation [6-11]. In this review, we will discuss the role of the GATA family of transcription factors in heart gene regulation and differentiation.

\section{Development of the chambers and the conduction system}

In vertebrates, the heart arises from cells in the anterior lateral plate mesoderm of the early embryo (stage E7-7.5 in mouse). By E7.5-8.0, during folding of the embryo and formation of the foregut, these cells migrate to the midline to form the primary heart tube. The embryonic myocardium of the tube is characterized by a primitive phenotype, i.e., limited cell proliferation, a poorly developed contractile apparatus, and slow conduction [12, 13]. During further development, the heart tube elongates by addition of progenitor cells to its poles. These cells are referred to as second heart field progenitors that are kept in an undifferentiated and rapidly proliferating state dorsal to the primitive heart tube $[14,15]$. Simultaneously, specific regions in the embryonic heart tube acquire a chamberspecific gene program, differentiate further, and expand by rapid proliferation to form the ventricular and atrial chamber myocardium ( $>$ E8.5). The chamber myocardium acquires properties of fast conduction, and the contractile apparatus starts to develop. In contrast, the regions flanking these differentiating chambers, the sinus venosus, the atrioventricular (AV) canal, and the outflow tract do not differentiate or expand and consequently form constrictions. The AV canal preserves characteristics of the embryonic myocardium of the tubular heart (Fig. 1), including its slow conductive property, ensuring AV delay

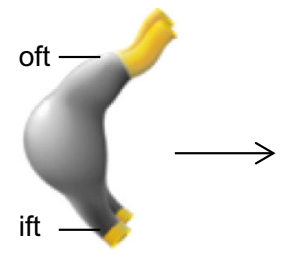

$E 8.5$

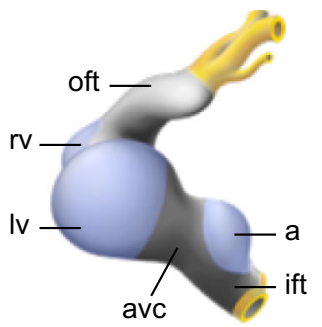

$E 9.5$
Fig. 1 Schematic overview of heart development. The early heart tube has an embryonic phenotype (gray). Chamber myocardium (blue) expands from the outer curvatures, whereas non-chamber myocardium (gray) of the inflow tract, atrioventricular canal, outflow of the impulse underlying the alternating contraction of the atria and ventricles. Signals from the AV canal are crucial for septation and valve formation, whereas the AV myocardium itself forms the AV node and AV ring bundles of the conduction system [16-18]. The fetal conduction system can be subdivided into more compartments such as the sinus node, the AV node, AV bundle, the left and right bundle branches, and the peripheral ventricular system. Each of these structures contains cardiomyocytes with pacemaker activity and specific properties that discriminate them from atrial and ventricular chamber myocardium.

Embryonic myocardial cells are confronted with a critical decision: differentiation into pacemaker-like cardiomyocytes of the AV canal or into working cardiomyocytes of the atrial and ventricular chambers. Bone morphogenetic protein 2 (Bmp2) is expressed in the primary heart tube where it stimulates the expression of T-box 2 (Tbx2), required for the development of the AV canal $[17,19,20]$. Both AV canal and chamber myocardium are competent to respond to BMP signaling [21, 22]. However, Bmp2 is selectively expressed in the AV canal myocardium precursors and conditional deletion of the BMP type I receptor ALK3 in the conduction system using specific Cre driver mice (cGata6-Cre) demonstrated that BMP signaling regulates the proper development of the $\mathrm{AV}$ conduction system [23, 24]. Consistently, although the downstream effector of BMP2, Smad4, is ubiquitously expressed at E10.5, its localization (and transcriptional activity) is predominantly nuclear in the AV canal [25, 26]. Interestingly, insertion of a transgenic construct harboring the AV canalspecific enhancer of the chicken Gata6 (cGata6) in the mouse genome leads to transgene activity in the cardiac crescent (E7.5) where both Bmp2 and $T b x 2$ are expressed $[27,28]$. These observations, together with functional and lineage tracing analyses for $T b x 2$, support the notion that the AV canal myocardial cells are already specified at this early stage $[18,29,30]$.

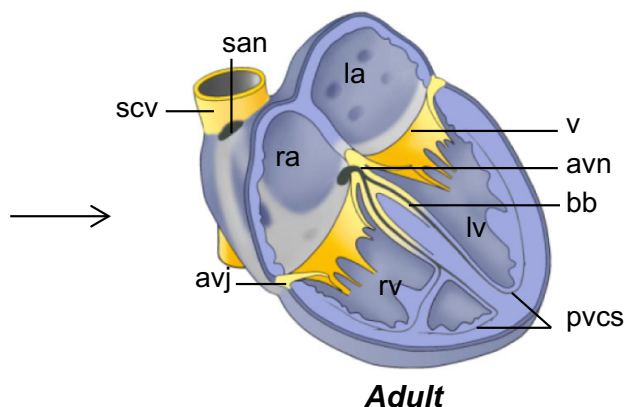

tract, and inner curvatures does not expand. $a$ atrium, $b b$ bundle branch, ift inflow tract, $l a$ left atrium, $l v$ left ventricle, $p v c s$ peripheral ventricular conduction system, $r a$ right atrium, $r v$ right ventricle, $s c v$ superior caval vein 
More broadly expressed cardiac transcription factors such as Tbx5, Tbx20, and Nkx2.5 are also required for the development of the conduction system [31, 32]. Chamber differentiation is suppressed by $\mathrm{Tbx} 2$ and $\mathrm{Tbx} 3$, which are selectively active in the AV canal myocardium where they suppress chamber-specific genes including Nppa (Anf, atrial natriuretic factor), Gjal ( $\mathrm{Cx} 43$, connexin 43), and Gja5 (Cx40, connexin 40) [33-35]. The sharp boundaries between these two compartments are obtained by repressive activities mediated by the Notch target genes Heyl and -2 [17, 36]. Heyl is expressed in the atria and Hey2 in the ventricles, but neither gene is found to be expressed in the AV canal myocardium [36, 37].

\section{Transcriptional activities of GATA factors in mammalian heart development}

The family of GATA transcription factors consists of six members, Gata1-6. In vertebrates, most tissues of either mesodermal or endodermal origin express one or more Gata4-6 factor during development. In the primitive streak, Gata4, -5, and -6 are detected in the precardiac mesoderm (stage E7.5) [38, 39]. The expression patterns of Gata 4 and -6 have been reported to be identical throughout the primary heart tube (E8.0-8.5). As the tube loops (E8.5), Gata5 becomes restricted to the endocardium, while Gata4 and -6 are maintained in the myocardium. Gata4 is detected in the endocardium of the atria and ventricles and endocardial cushions of the AV canal and outflow tract. Gata4 and -6 are expressed in both the AV canal and chamber myocardium, with no evidence for differential expression levels and subcellular compartmentalization [38, 40-43]. Since the spatial organization of the nucleus may impact on activation or repression of gene expression and interaction with co-factors, assessment of the localization of GATA factors within the nucleus might be relevant.

Mice null for Gata4 die between E8 and 9 because of defects in heart morphogenesis and ventral closure of the foregut. Aberrant heart formation in these mice is mainly secondary to defects in the extra-embryonic and definitive endoderm that affects migration and folding morphogenesis of the precardiogenic splanchnic mesodermal cells [44-46]. Using a tetraploid embryo complementation strategy, it has been possible to show that Gata4 is cell autonomously required for cardiogenesis [47]. Elegant functional analyses utilizing knockout ES cells and tissuespecific disruption in mice unequivocally established a role for Gata4, -5 , and -6 in controlling heart development that is consistent with their expression pattern. Studies using heterozygous Gata4 and Gata6 mutants demonstrated that both factors are critical for general cellular processes including cardiac cell commitment, differentiation, proliferation, and survival, hampering the analysis of their function in the formation of structures that arise later [9, 11, 48]. The levels of Gata4 and -6 are critical for normal embryonic development and survival $[9,11,48]$. Embryos in which the expression of Gata4 protein in the myocardium is reduced by $50 \%$ survive. In contrast, a reduction of $70 \%$ causes fetal death between E13.5 and 16.5. These fetuses have AV canal defects, double outlet right ventricle, and hypoplastic ventricular myocardium. GATA6 mutations cause persistent truncus arteriosus in human [49]. Gata6 also regulates morphogenetic patterning of the cardiac aortic arch [50]. Wnt2, a ligand expressed specifically in the cardiac inflow tract, acts with Gata6 to regulate the development of the atrial myocardium, pulmonary veins, and AV canal [51]. Gata6 is downregulated in Gata4 null mice and Gata6 null embryos show down regulation of Gata4, indicating that these factors cross regulate each other [44]. In addition, Gata6 compensates the loss of Gata4 in inducing the myocardial gene program [39, 48]. This transcriptional interconnection is consistent with the observation that Gata4 and Gata6 double heterozygote mice die very early during embryonic development [48]. Gata4 and Gata6 mutants show defects of the venous pole region, suggesting involvement of these factors in embryonic inflow tract development and the venous blood connection [52]. Gata5 interacts with Gata4 and -6 in endocardial cushion formation, i.e., morphogenesis of the atrioventricular valves and outflow tract [53].

Gata4 is known to activate many cardiac genes displaying regionally restricted expression, including the chamber-specific genes Nppa and Nppb (Bnf, brain natriuretic factor) [54] and the contractile genes $M y h 6$ ( $\alpha$-MHC) and $M y h 7$ ( $\beta$-MHC) [55, 56]. Gata4 is shown to activate the gene Gja5 (Cx40) [57] that plays an important role in cell-cell communication in cardiomyocytes of the atrial chambers and in the ventricular conduction system [58]. Gata6 activates the ion exchanger gene Ncxl [59] and the potassium channel gene $K v 4.2$ [60]. Expression of $N k x 2.5$, one of the earliest markers of the cardiac lineage in vertebrates, depends on GATA binding sites [61]. GATA factors also control the expression of $M e f c 2 c$ in outflow tract and right ventricular myocardium [62]. Gata4 is a direct transcriptional activator of cell cycle genes cyclin D2 and $C d k 4$. Gata4 physically and functionally interacts with CDK4 [63] and is required for cardiomyocyte proliferation [64]. Gata4 chromatin occupancy changes markedly between fetal and adult heart, with only partial overlap in binding sites [65]. This underlies the importance of rating the quality of ChIP-seq experiments and the datasets generated in order to preclude incomparability due to low quality. 


\section{Interplay between GATA factors and chromatin}

Approximately seven million GATA motifs are present in the human genome [11]. This raises the question of which motifs are occupied by Gata4 in vivo. With the development of ChIPseq assays to assess Gata4 chromatin occupancy genomewide, it has become clear that only a small subset of the GATA motifs are occupied in heart tissue, suggesting the existence of a GATA recognition code that dictates site occupancy [6668]. How GATA factors discriminate among these motifs in a time- and location-dependent manner is unclear [69]. This question directly applies to Gata4, and -6 in heart development. An important consideration is whether Gata4 and Gata6 occupy identical or different WGATAR motifs in different myocardial compartments. Since Gata4 and -6 have redundant functions in regulating myocardial genes, addressing these questions might well explain their possible similar participation in the conduction system and chamber gene programs. Local chromatin environment, nearest neighboring factor binding motifs and intrinsic features of the WGATAR motifs are likely important parameters underlying the GATA recognition code. The transcriptional activation domains of Gata4, -5, and -6 are partially conserved, suggesting a similar mechanism of transcriptional activation within this Gata subfamily. Although Gata4, -5 , and -6 bind a GATA or GATA-like sequence element, their individual affinities for various regulatory elements might also depend on flanking nucleotide sequences or on interactions with co-factors and other transcription factors. Gata4 ChIP-seq analyses also revealed that a large fraction of genomic regions occupied by Gata4 are not associated with a recognizable GATA binding site, indicating indirect binding [66-68]. Consistent with this, in vitro reporter assays suggest that Gata4 transcriptional activities do not necessarily require Gata4 DNA binding [11]. Since ChIP assesses protein-DNA proximity by cross linking and not by direct binding, it would be interesting to verify GATA binding using in vivo foot printing. Assessment of the genomic distribution of Gata4 and Gata6 occupancy by cell sorted-based genome and proteome-wide technologies should facilitate our understanding of how GATA factors select chromatin target sites. This has become a realistic option with the development of protocols to perform genome-wide analysis on small amounts of tissue [70-72].

There is no doubt that emerging molecular technologies will help in understanding the function of GATA TFs in cardiac lineage specification. GATA TFs regulate transcription during differentiation by mediating long-range DNA looping. Whether this is the case in the context of heart development is unknown. Chromosome conformation capture technologies (e.g., 3-5C, Hi-C) were developed to identify long-range chromatin interactions [73]. The ENCODE project has provided access to valuable data on genome-wide chromatin occupancy of transcription factors, chromatin-modifying and -remodeling enzymes, and histone modifications in heart tissues [74]. Merging these datasets can further facilitate identification of GATA regulatory elements. The functional importance of GATA elements can be assessed in vivo using recent genome editing technologies [75]. Recent ChIP-seq analyses show that in the context of cardiac stress, Gata4 binds to a subset of fetal genes such as $N p p b$ [76] leading to the reactivation of the "fetal gene program." However, reactivation of fetal genes constitutes a small portion of the transcriptional response of the diseased adult heart [65]. ChIP-seq and motif analysis have shown that the GATA motif is highly overrepresented in Gata4-bound regions from fetal, adult, and disease heart [65]. These analyses also indicate that the selection of GATA binding sites is influenced by the interaction of Gata4 with other TFs. The co-enriched motifs of other TFs are rather distinct between fetal-specific and adult-specific Gata4-bound regions. These changes are associated with altered expression of transcriptional regulators between fetal and adult heart. Interestingly, in hypertrophic hearts, Gata4 regions contain an atypical GATA motif and are enriched for the NFAT binding motif. NFATs are essential mediators of cardiac hypertrophy.

From studies in cell culture, two distinct DNA binding modes of GATA transcription factors have been deduced [77]. In the wrapping mode, the two zinc fingers of a GATA factor wrap around a palindromic GATA site, cooperatively enhancing binding affinity and kinetic stability. In the bridging mode, a single GATA DNA binding domain bridges two pieces of DNA. Because the DNA binding domains of GATA proteins are highly conserved, these structural features could be shared by all six GATA family members. This may have important implications for transcriptional regulation by GATA proteins. For example, varying concentrations of GATA proteins during heart development may not just affect their occupancy of DNA but also could switch their DNA binding mode and affect transcriptional networks. GATA proteins may loop DNA through co-factors (e.g., Fog proteins) [78], which have also been shown to play important roles in mediating DNA loop formation [79-81].

Mechanisms underlying the function of governing the decision of whether GATA TFs function as activators or repressors of a targets gene have been studied in depth in the context of the hematopoietic system. Using the aforementioned approaches, it has been shown that in this lineage context, Gata1 uses Fog1 to activate and or repress the majority of its target genes [82, 83]. Fog1 associates with the repressive nucleosome remodeling and deacetylase NuRD complex [84]. The transcription factor Gata1 is required for terminal erythroid maturation and functions as an activator 
or repressor depending on gene context [85]. During late erythroid development, PRC2 complex is involved in late stages of silencing of some Gatal repressed genes. The Gata1-interacting coregulator Friend of Gata-1 (Fog1) mediates Gata1-dependent activation and repression in a context-dependent manner [82, 83]. Fog1 facilitates Gata1 chromatin occupancy and interacts with the repressive nucleosome remodeling and deacetylase (NuRD) complex [84]. GATA TFs in the context of the hematopoietic system are known to cooperate with Brahma-related gene-1 (BRG1), an enzyme that disrupts chromatin structure, acting as either an activator or a repressor in the SWI-SNF nucleosome remodeling complex $[86,87]$. BRG1 could be a privileged mediator or partner in the action of cardiac GATA factors on chromatin structure.

Chromatin remodeling enzymes regulate Gata4 target genes by post-translational modifications of Gata4 [11, 88]. Gata4 physically interacts with histone acetyl transferases (HATs) and histone deacetylases (HDACs) [89-91]. Activation of Gata4 is mediated through its acetylation by the HAT p300 [92] which is consistent with the large proportion of cardiac enhancers occupied by both [43, 65]. In turn, p300 also acetylates histones such as the histone 3 at lysine 27 (H3K27ac), a mark associated with active enhancers [93]. Gata4 cooperates with the acetylase p300 to deposit $\mathrm{H} 3 \mathrm{~K} 27 \mathrm{ac}$ at cardiac enhancers to stimulate transcription [65]. Paradoxically, p300 occupies both GATA-activated and -repressed genes [94, 95]. A large portion of Gata4 occupancy occurs at sites that have a closed chromatin configuration in the normal heart [65]. HDAC1 and HDAC2 can physically interact with Gata4 [89-91] and are functionally redundant in the heart [96]. Gata4 forms a complex with Hopx which serves as an adapter to facilitate HDAC2 recruitment, which in turn reduces Gata4 activity by deacetylation [88]. The activity of GATA factors can also be regulated through methylation. The polycomb-repressive complex 2 (PRC2) directly binds and methylates Gata4, reducing its interaction with and acetylation by the HAT p300 [97]. This results in attenuation of Gata4 transcriptional activity [97]. Given the interplay between GATA factors and multiple coregulators, a system biology approach will be required to develop a comprehensive model for the cardiac transcriptional networks around the GATA factors.

\section{GATA factors interact with other heart-expressed transcription factors}

GATA factors have a dual finger module which is highly conserved among the six mammalian proteins. The C-terminal finger mediates sequence-specific DNA binding, while the N-terminal finger modulates DNA binding and contacts DNA. The zinc fingers interact with multiple coregulators. Disruption of these interactions has been reported to underlie congenital heart defects [6]. A precise balance between interactions with co-factors appears essential for the regulation of their spatial and temporal activity. It was previously demonstrated by in vitro and in vivo studies that Gata4 and Tbx5 physically interact and cooperatively activate endocardial [98] and myocardial genes such as Nppa [6]. Gata6 and Tbx5 genetically interact in vivo [40]. Combinatorial expression of Gata4, $\mathrm{Nkx} 2.5$, and SRF (serum response factor) directs early cardiac gene activity $[66,68,99]$. Although the Gata4 and Gata6 protein interaction with the primary myocardium/conduction system-specific protein Tbx 3 has not been firmly established, ChIP-seq data for Gata4 and Tbx3 from heart tissue revealed a large portion of overlapping peaks, suggesting that $\mathrm{Tbx} 3$ and Gata4 might cooperatively repress the working myocardial gene program [67]. Gata6 regulates transcription of $I d 2$ [100], a transcriptional repressor involved in conduction system lineage specification [31]. Gata4 and Smad4 form proteinprotein interactions and cooperatively regulate cardiac valve development by regulating Id2 [101]. Further studies will reveal whether Gata4 also activates $I d 2$ in the conduction system and whether a Gata4/6-Smad4-Id2 network module participates in the normal control of conduction system gene expression and specification. Studies in postnatal cardiomyocytes demonstrated that Gata4 and Gata6 can functionally interact with each other, at a single GATA element, to synergistically activate the Nppa and Nppb promoters [102]. Retinoic acid receptors regulate Nppa and $N p p b$ genes via direct interaction with Gata4 and its corepressor, Fog2 [103]. Mutations of Gata4 in human and in mouse models can result in abrogation of physical interaction with some of these co-factors [104].

\section{GATA factors and conduction system-specific enhancers}

DNA elements conferring cell-type-specific gene expression are ideal to analyze the molecular mechanisms that underlie the localized gene expression of conduction system genes. For example, one of the enhancers that flank the cGata6 gene directs transgene expression throughout the entire primary heart tube and in all regions of primary myocardium that persist at later stages (Fig. 2) [27, 43, 105]. Interestingly, this enhancer module is completely inactivated in newly arising chamber myocardium. Several other DNA elements conferring AV-restricted patterns of gene activity have been identified in mouse transgenic embryos, enhancers regulating Tbx2 [106], Tbx3 [107], Gjd3 (Cx30.2, connexin 30.2) involved in conduction slowing [41], and the promoter of $c T n I$ [108]. Interestingly, the common features of these AV 


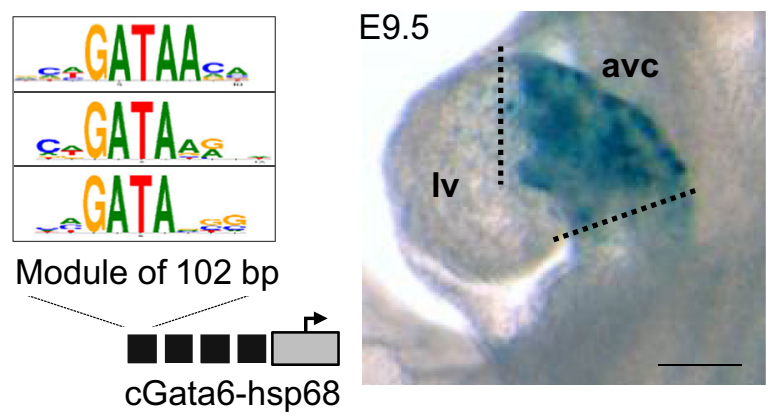

Fig. 2 Tandemly repeated copies of a 102 bp cGata6 enhancer fragment drive expression in the developing AV canal at stage E9.5; a module of 102 bp contains 3 GATA binding sites. Scale bars $100 \mu \mathrm{m}$

canal-specific regulatory elements are the presence of GATA consensus sequences and in vivo Gata4 occupancy as demonstrated by recent ChIP studies [65, 67]. Moreover, mutations in Gata4 and - 6 are responsible for congenital heart defects including AV canal defects and arrhythmias in human and mouse $[8,11,41,109]$, implicating their function in conduction system tissues [100, 110-113]. Conduction system-restricted gene activity is controlled by more broadly expressed transcription factors. We and others have shown that the activity of these AV canal-specific regulatory elements depend on GATA binding sites [41, 43, 105]. The activity of the Gjd3-AV canal enhancer also depends on the broadly expressed factor Tbx5 [41]. Gata4, Tbx5, and $\mathrm{Nkx} 2.5$ are expressed in largely overlapping domains in the conduction system and co-occupy several conduction system gene loci [67]. This suggests that they may cooperatively activate genes in the conduction system [114]. Despite widespread expression throughout the myocardium, these factors have a specific role in patterning the $\mathrm{AV}$ conduction system.

\section{How do GATA factors acquire their AV canal- specific functions?}

The emergence of cell sorting-based transcriptomics and ChIP-seq is contributing to a better understanding on how GATA factors acquire specific functions. By integrating a variety of ChIP-seq datasets, we found that Gata4 binds to loci associated with both active and repressive chromatin signatures [43]. We tested whether the AV canal-specificity of these enhancers involves a mechanism of repression outside the AV canal. The promoter of the rat cardiac troponin $\mathrm{T}$ (cTnT, Tnnt2) gene drives efficient pan-cardiac expression in transgenic mice [115]. Previously identified AV canal enhancers were coupled to the $c \operatorname{TnT}$-lacZ transgenic construct, and a repressive function was assayed in mouse transgenic embryos.
Analysis of ChIP-seq data from tissue-specific compartments coupled with validation in transgenics revealed these AV canal enhancers act as cell-type-specific switches, activating transcription in the AV canal and repressing transcription in the chambers. We found that mutation of the GATA binding sites affected the repressive activity of the cGata6 and Tbx2 enhancers. Thus, GATAdependent AV canal-specific enhancers mediate both region-dependent gene activation and repression. Gata4 activates such enhancers in synergy with Bmp2/Smad signaling. Our data indicated that GATA sites within AV canal-specific regulatory regions recruit a Gata4-Smad4HAT p300 transcriptional activation complex. This is associated with AV canal-specific acetylation of histone H3K27.

In chamber myocardium, Gata4 represses these enhancers through pan-cardiac HDACs and chamber-specific Notch target genes Hey1 and -2 [43]. The cGata6, Gjd3, and $c T n I$ modules do not contain a consensus Hey binding site (i.e., E-box), suggesting that Hey factors mediate repression via GATA protein interaction [116]. We speculate that Gata4-HDAC mediated deacetylation of H3K27 serves as a mode of confinement of the conduction system gene program, preventing its activity in the chambers. Interestingly, histological analysis revealed that $\mathrm{Tbx} 2$ expression was only slightly expanded in Hey 1 and Hey2 double-deficient mice [117]. This implies that Hey1 and -2 refine the boundary between the AV canal and the chambers by repressing $T b x 2$ [36]. These factors contribute but are not essential for chamber repression by Gata4-HDACs.

GATA sites in the AV canal regulatory elements thus serve as a platform to recruit broadly active histone modification enzymes and localized co-factors to drive AV canal-specific gene activity (Fig. 3). Obviously, posttranslational modifications could play a role in this process such as direct acetylation/deacetylation of Gata4, -6 proteins accompanied by methylation by the aforementioned

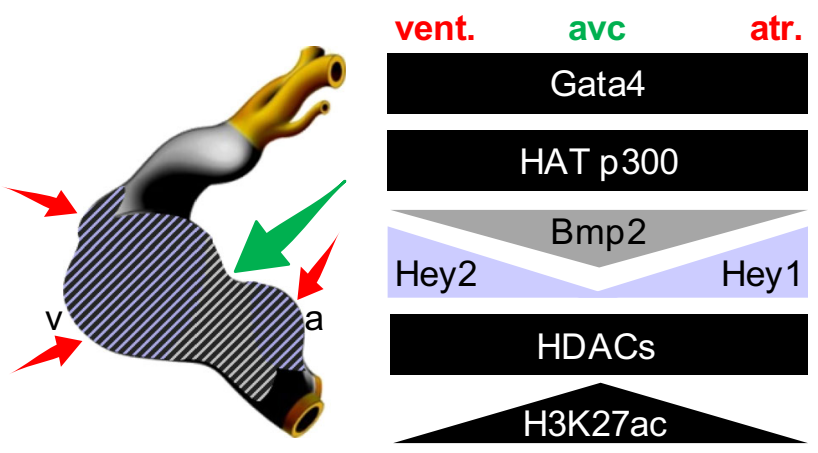

Fig. 3 Cartoon depicting the overlapping expression patterns of Gata4, broadly active histone modification enzymes, localized cofactors, and the level of $\mathrm{H} 3 \mathrm{~K} 27 \mathrm{ac}$ enrichment on AV canal gene loci at stage E9.5-E10.5, atr atria, vent ventricles 
PRC2 complex. Whether a change of GATA factor concentrations guide this dual function is unknown. Since inspection of Gata4, Bmp2, and cGata6-lacZ expression patterns revealed that they are already expressed in the cardiac crescent, it suggests that already at the primitive streak stage, the GATA-Smad-HAT complex may occupy primary myocardial gene loci. This could lead to acetylation of $\mathrm{H} 3 \mathrm{~K} 27$ at this early stage. Subsequently upon chamber formation, these loci may become deacetylated in cells receiving appropriate local guidance cues.

\section{Perspectives on the requirement of GATA factors to reprogram pacemaker cell identity}

Disease, aging, or gene defects may cause conduction system dysfunction, a common cardiac disorder that currently requires the implantation of an electronic pacemaker. Recent advances in the stem cell field indicate that the creation of induced pacemaker cells may offer a promising alternative to repair diseased pacemaker myocardium [118]. Developmental and genetic studies have identified several cardiac transcription factors that are necessary for the development of the conduction system [13]. Currently, there is interest in using this information to reprogram cells to pacemaker cells by transducing TF genes [119]. In vitro direct reprogramming of human fibroblasts toward a cardiomyocyte-like cell type requires a minimal cocktail of transcription factors that include Gata4 [120, 121]. However, in vitro reprogramming remains inefficient and better understanding of epigenetic changes associated with TF overexpression may provide clues for improving the efficiency [122]. A key question is how factors such as Gata4 could enable cellular reprogramming. Gata4 occupancy does not necessarily imply that it activates transcription. Nevertheless, its occupancy seems to initiate a series of changes that allow the enhancer to recruit its full set of transcription factors and chromatin remodeling complexes. At a particular stage in differentiation, some transcription factors, defined as "pioneer factors," are able to access DNA target sites in chromatin where other factors are not yet able [123]. These factors can recruit chromatin modifiers and activate or repress transcription. Similarly, Gata4 has been proposed to initiate formation of transcriptional regulatory complexes on closed chromatin [124]. Later during differentiation and depending on the context such as the presence of binding sites for other transcription factors, an enhancer may become associated with active histone marks such as the $\mathrm{H} 3 \mathrm{~K} 27 \mathrm{ac}$ or with repressive marks and the poised RNA pol II complex. As development continues, we and others have shown that the binding of additional factors or changes in cofactor availability may change the activity of the targeted element. This change of activity occurs when the binding of the priming factor is maintained which enables a rapid transcriptional response to inductive signals. In the lineage conversion of one cell type to another, overexpression of GATA factors may actively open-up the local chromatin and make it competent for other factors to bind.

In many of the reprogramming studies, multiple factors are required for the cellular conversion, emphasizing the role of cooperativity among factors in the starting population of cells. Binding cooperatively could be sufficient to simultaneously engage a target site in chromatin and activate gene expression. Stoichiometry of Gata4 and its cofactors influences the efficiency and quality of cardiomyocyte reprogramming [125]. Although the methods using this cocktail of cardiac transcription factors provided the proof of concept that somatic cells can be reprogrammed into cardiomyocytes, reprogramming toward specific human cardiac subtypes is a remaining challenge. Moreover, only a small subset of the targeted cells becomes

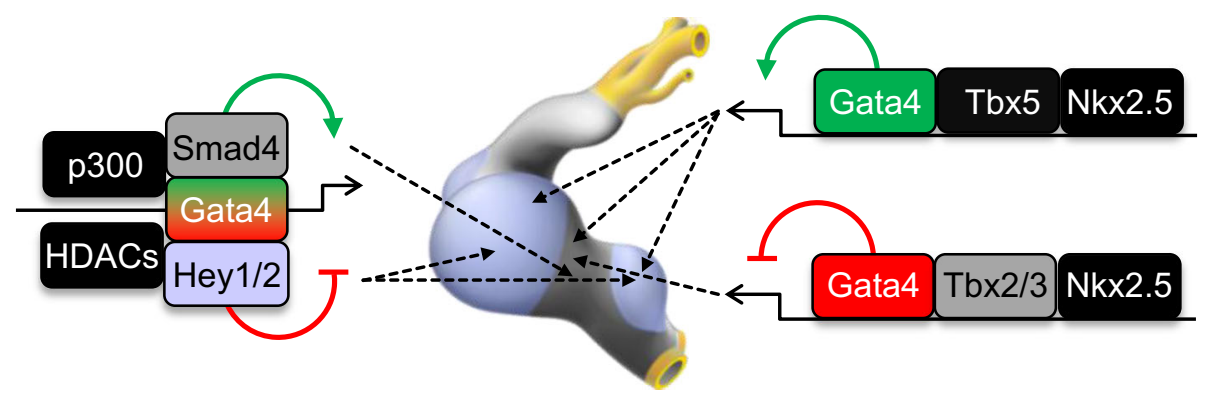

Fig. 4 Working model of a GATA factor regulatory network for primary versus chamber myocardium formation. GATA elements direct complex formation of cardiac transcription factors, broadly active histone modification enzymes, and localized co-factors to drive specific gene activity. GATA and other broadly expressed transcription factors cooperatively activate chamber genes in the chambers and AV canal genes in the AV canal. Locally expressed Tbx2/3 and GATA factors cooperatively repress the working myocardial gene program in the AV canal/primary myocardium. GATA binding site enhancers recruit a Gata4/Smad4/HATp300 transcriptional activation complex in the AV canal and a Gata4/Hey1,2/HDACs transcriptional repression complex in the chambers, which coordinately establish AV canal-specific gene expression. Broadly expressed, primary- and chamber-specific transcription factors are depicted in black, gray, and blue, respectively 
reprogrammed in vitro. Inhibitors of histone-modifying enzymes are known to enhance reprogramming, suggesting that modulation of these chromatin-modifying enzymes may be exploited to more efficiently generate induced pacemaker cells [126-128]. In this regard, it remains to be determined whether manipulating the GATA-Smad-HAT/ GATA-Hey-HDAC complexes could target many silent conduction system-specific sites, open the chromatin for active transcription and enhance the reprogramming toward human pacemaker cells efficiently.

In summary, the cooperation of GATA factors with active histone modification enzymes and localized co-factors ensures proper spatial-temporal expression of cardiac genes during normal embryonic development (Fig. 4). Defining how GATA or GATA-co-factors act in different myocardial compartments to enable epigenetic regulatory events will provide insight into the biology of progenitor cells leading to methods for increasing the efficiency of directed differentiation of pluripotent cells and cellular reprogramming into myocardial subtypes. Addressing this question is also critical for understanding the origin of congenital heart defects.

Acknowledgments S. Stefanovic was supported by post-doctoral awards from EMBO and the ESC. V. M. Christoffels was supported by the ZonMw TOP 912-12-086.

Open Access This article is distributed under the terms of the Creative Commons Attribution 4.0 International License (http://creativecommons.org/licenses/by/4.0/), which permits unrestricted use, distribution, and reproduction in any medium, provided you give appropriate credit to the original author(s) and the source, provide a link to the Creative Commons license, and indicate if changes were made.

\section{References}

1. Bruneau BG (2008) The developmental genetics of congenital heart disease. Nature 451(7181):943-948

2. Fahed AC et al (2013) Genetics of congenital heart disease: the glass half empty. Circ Res 112(4):707-720

3. Vissers LE et al (2004) Mutations in a new member of the chromodomain gene family cause CHARGE syndrome. Nat Genet 36(9):955-957

4. Miyake $\mathrm{N}$ et al (2013) KDM6A point mutations cause Kabuki syndrome. Hum Mutat 34(1):108-110

5. Zaidi $S$ et al (2013) De novo mutations in histone-modifying genes in congenital heart disease. Nature 498(7453):220-223

6. Garg V et al (2003) GATA4 mutations cause human congenital heart defects and reveal an interaction with TBX5. Nature 424(6947):443-447

7. Hirayama-Yamada K et al (2005) Phenotypes with GATA4 or NKX2.5 mutations in familial atrial septal defect. Am J Med Genet A 135(1):47-52

8. Lin X et al (2010) A novel GATA6 mutation in patients with tetralogy of Fallot or atrial septal defect. J Hum Genet 55(10):662-667
9. Pu WT et al (2004) GATA4 is a dosage-sensitive regulator of cardiac morphogenesis. Dev Biol 275(1):235-244

10. Mohan RA et al (2014) A mutation in the Kozak sequence of GATA4 hampers translation in a family with atrial septal defects. Am J Med Genet A 164A(11):2732-2738

11. Zhou P, He A, Pu WT (2012) Regulation of GATA4 transcriptional activity in cardiovascular development and disease. Curr Top Dev Biol 100:143-169

12. Moorman AF, Christoffels VM (2003) Cardiac chamber formation: development, genes, and evolution. Physiol Rev 83(4):1223-1267

13. Christoffels VM et al (2010) Development of the pacemaker tissues of the heart. Circ Res 106(2):240-254

14. Buckingham M, Meilhac S, Zaffran S (2005) Building the mammalian heart from two sources of myocardial cells. Nat Rev Genet 6(11):826-835

15. Zaffran S, Kelly RG (2012) New developments in the second heart field. Differentiation 84(1):17-24

16. Armstrong EJ, Bischoff J (2004) Heart valve development: endothelial cell signaling and differentiation. Circ Res 95(5):459-470

17. Ma L et al (2005) Bmp2 is essential for cardiac cushion epithelial-mesenchymal transition and myocardial patterning. Development 132(24):5601-5611

18. Aanhaanen WT et al (2010) Developmental origin, growth, and three-dimensional architecture of the atrioventricular conduction axis of the mouse heart. Circ Res 107(6):728-736

19. Singh MK et al (2005) Tbx20 is essential for cardiac chamber differentiation and repression of Tbx2. Development 132(12):2697-2707

20. Singh R et al (2012) Tbx2 and Tbx3 induce atrioventricular myocardial development and endocardial cushion formation. Cell Mol Life Sci 69(8):1377-1389

21. Huang J et al (2012) Myocardin regulates BMP10 expression and is required for heart development. J Clin Invest 122(10):3678-3691

22. Chen $\mathrm{H}$ et al (2004) BMP10 is essential for maintaining cardiac growth during murine cardiogenesis. Development 131(9):2219-2231

23. Stroud DM et al (2007) Abnormal conduction and morphology in the atrioventricular node of mice with atrioventricular canal targeted deletion of Alk3/Bmprla receptor. Circulation 116(22):2535-2543

24. Anderson RH et al (2008) Letter by Anderson et al regarding article, "Abnormal conduction and morphology in the atrioventricular node of mice with atrioventricular canal-targeted deletion of Alk3/Bmpr1a receptor". Circulation 118(6):e105 (author reply e107)

25. Flanders KC, Kim ES, Roberts AB (2001) Immunohistochemical expression of Smads 1-6 in the 15-day gestation mouse embryo: signaling by BMPs and TGF-betas. Dev Dyn 220(2):141-154

26. Monteiro RM et al (2008) Real time monitoring of BMP Smads transcriptional activity during mouse development. Genesis 46(7):335-346

27. Davis DL et al (2001) A GATA-6 gene heart-region-specific enhancer provides a novel means to mark and probe a discrete component of the mouse cardiac conduction system. Mech Dev 108(1-2): 105-119

28. Yamada M et al (2000) Expression of chick Tbx-2, Tbx-3, and Tbx-5 genes during early heart development: evidence for BMP2 induction of Tbx2. Dev Biol 228(1):95-105

29. Aanhaanen WT, Moorman AF, Christoffels VM (2011) Origin and development of the atrioventricular myocardial lineage: insight into the development of accessory pathways. Birth Defects Res A Clin Mol Teratol 91(6):565-577 
30. Aanhaanen WT et al (2009) The Tbx2+ primary myocardium of the atrioventricular canal forms the atrioventricular node and the base of the left ventricle. Circ Res 104(11):1267-1274

31. Moskowitz IP et al (2007) A molecular pathway including Id2, Tbx5, and Nk2-5 required for cardiac conduction system development. Cell 129(7):1365-1376

32. Risebro CA et al (2012) Epistatic rescue of Nkx2.5 adult cardiac conduction disease phenotypes by prospero-related homeobox protein 1 and HDAC3. Circ Res 111(2):e19-e31

33. Hoogaars WM et al (2004) The transcriptional repressor Tbx3 delineates the developing central conduction system of the heart. Cardiovasc Res 62(3):489-499

34. Christoffels VM et al (2004) T-box transcription factor Tbx2 represses differentiation and formation of the cardiac chambers. Dev Dyn 229(4):763-770

35. Harrelson $Z$ et al (2004) Tbx2 is essential for patterning the atrioventricular canal and for morphogenesis of the outflow tract during heart development. Development 131(20):5041-5052

36. Rutenberg JB et al (2006) Developmental patterning of the cardiac atrioventricular canal by Notch and Hairy-related transcription factors. Development 133(21):4381-4390

37. Fischer A et al (2007) Combined loss of Heyl and HeyL causes congenital heart defects because of impaired epithelial to mesenchymal transition. Circ Res 100(6):856-863

38. Peterkin $T$ et al (2005) The roles of GATA-4, -5 and -6 in vertebrate heart development. Semin Cell Dev Biol 16(1):83-94

39. Peterkin T, Gibson A, Patient R (2007) Redundancy and evolution of GATA factor requirements in development of the myocardium. Dev Biol 311(2):623-635

40. Maitra M et al (2009) Interaction of Gata4 and Gata6 with Tbx5 is critical for normal cardiac development. Dev Biol 326(2):368-377

41. Munshi NV et al (2009) Cx30.2 enhancer analysis identifies Gata4 as a novel regulator of atrioventricular delay. Development 136(15):2665-2674

42. Burch JB (2005) Regulation of GATA gene expression during vertebrate development. Semin Cell Dev Biol 16(1):71-81

43. Stefanovic S et al (2014) GATA-dependent regulatory switches establish atrioventricular canal specificity during heart development. Nat Commun 5:3680

44. Molkentin JD et al (1997) Requirement of the transcription factor GATA4 for heart tube formation and ventral morphogenesis. Genes Dev 11(8):1061-1072

45. Molkentin JD et al (2000) Direct activation of a GATA6 cardiac enhancer by $\mathrm{Nkx} 2.5$ : evidence for a reinforcing regulatory network of Nkx2.5 and GATA transcription factors in the developing heart. Dev Biol 217(2):301-309

46. Kuo CT et al (1997) GATA4 transcription factor is required for ventral morphogenesis and heart tube formation. Genes Dev 11(8): 1048-1060

47. Zhao R et al (2008) Loss of both GATA4 and GATA6 blocks cardiac myocyte differentiation and results in acardia in mice. Dev Biol 317(2):614-619

48. Xin $M$ et al (2006) A threshold of GATA4 and GATA6 expression is required for cardiovascular development. Proc Natl Acad Sci USA 103(30):11189-11194

49. Kodo K et al (2009) GATA6 mutations cause human cardiac outflow tract defects by disrupting semaphorin-plexin signaling. Proc Natl Acad Sci USA 106(33):13933-13938

50. Lepore JJ et al (2006) GATA-6 regulates semaphorin 3C and is required in cardiac neural crest for cardiovascular morphogenesis. J Clin Invest 116(4):929-939

51. Tian Y et al (2010) Characterization and in vivo pharmacological rescue of a Wnt2-Gata6 pathway required for cardiac inflow tract development. Dev Cell 18(2):275-287
52. Watt AJ et al (2004) GATA4 is essential for formation of the proepicardium and regulates cardiogenesis. Proc Natl Acad Sci USA 101(34):12573-12578

53. Laforest B, Nemer M (2011) GATA5 interacts with GATA4 and GATA6 in outflow tract development. Dev Biol 358(2):368-378

54. McBride K, Nemer M (2001) Regulation of the ANF and BNP promoters by GATA factors: lessons learned for cardiac transcription. Can J Physiol Pharmacol 79(8):673-681

55. Molkentin JD, Kalvakolanu DV, Markham BE (1994) Transcription factor GATA-4 regulates cardiac muscle-specific expression of the alpha-myosin heavy-chain gene. Mol Cell Biol 14(7):4947-4957

56. Wang GF et al (1998) A positive GATA element and a negative vitamin D receptor-like element control atrial chamber-specific expression of a slow myosin heavy-chain gene during cardiac morphogenesis. Mol Cell Biol 18(10):6023-6034

57. Linhares VL et al (2004) Transcriptional regulation of the murine Connexin 40 promoter by cardiac factors Nk2-5, GATA4 and Tbx5. Cardiovasc Res 64(3):402-411

58. Beyer S, Kelly RG, Miquerol L (2011) Inducible Cx40-Cre expression in the cardiac conduction system and arterial endothelial cells. Genesis 49(2):83-91

59. Koban MU et al (2001) A distant upstream region of the rat multipartite $\mathrm{Na}(+)-\mathrm{Ca}(2+)$ exchanger $\mathrm{NCX} 1$ gene promoter is sufficient to confer cardiac-specific expression. Mech Dev 109(2):267-279

60. Jia Y, Takimoto K (2003) GATA and FOG2 transcription factors differentially regulate the promoter for $\mathrm{Kv} 4.2 \mathrm{~K}(+)$ channel gene in cardiac myocytes and PC12 cells. Cardiovasc Res 60(2):278-287

61. Searcy RD et al (1998) A GATA-dependent nkx-2.5 regulatory element activates early cardiac gene expression in transgenic mice. Development 125(22):4461-4470

62. Dodou E et al (2004) Mef2c is a direct transcriptional target of ISL1 and GATA factors in the anterior heart field during mouse embryonic development. Development 131(16):3931-3942

63. Gallagher JM et al (2014) Carboxy terminus of GATA4 transcription factor is required for its cardiogenic activity and interaction with CDK4. Mech Dev 134:31-41

64. Rojas A et al (2008) GATA4 is a direct transcriptional activator of cyclin D2 and Cdk4 and is required for cardiomyocyte proliferation in anterior heart field-derived myocardium. Mol Cell Biol 28(17):5420-5431

65. He A et al (2014) Dynamic GATA4 enhancers shape the chromatin landscape central to heart development and disease. Nat Commun 5:4907

66. He A et al (2011) Co-occupancy by multiple cardiac transcription factors identifies transcriptional enhancers active in heart. Proc Natl Acad Sci USA 108(14):5632-5637

67. van den Boogaard $M$ et al (2012) Genetic variation in T-box binding element functionally affects SCN5A/SCN10A enhancer. J Clin Invest 122(7):2519-2530

68. Schlesinger $\mathbf{J}$ et al (2011) The cardiac transcription network modulated by Gata4, Mef2a, Nkx2.5, Srf, histone modifications, and microRNAs. PLoS Genet 7(2):e1001313

69. Bresnick EH et al (2005) Developmental control via GATA factor interplay at chromatin domains. J Cell Physiol 205(1):1-9

70. Bonn S et al (2012) Cell type-specific chromatin immunoprecipitation from multicellular complex samples using BiTS-ChIP. Nat Protoc 7(5):978-994

71. Adli M, Bernstein BE (2011) Whole-genome chromatin profiling from limited numbers of cells using nano-ChIP-seq. Nat Protoc 6(10): 1656-1668 
72. Shankaranarayanan P et al (2012) Single-tube linear DNA amplification for genome-wide studies using a few thousand cells. Nat Protoc 7(2):328-338

73. de Laat W, Duboule D (2013) Topology of mammalian developmental enhancers and their regulatory landscapes. Nature 502(7472):499-506

74. ENCODE Project Consortium (2004) The ENCODE (ENCyclopedia of DNA elements) project. Science 306(5696):636-640

75. Harrison MM et al (2014) A CRISPR view of development. Genes Dev 28(17):1859-1872

76. Molkentin JD et al (1998) A calcineurin-dependent transcriptional pathway for cardiac hypertrophy. Cell 93(2):215-228

77. Chen Y et al (2012) DNA binding by GATA transcription factor suggests mechanisms of DNA looping and long-range gene regulation. Cell Rep 2(5):1197-1206

78. Jing $\mathrm{H}$ et al (2008) Exchange of GATA factors mediates transitions in looped chromatin organization at a developmentally regulated gene locus. Mol Cell 29(2):232-242

79. Chlon TM, Crispino JD (2012) Combinatorial regulation of tissue specification by GATA and FOG factors. Development 139(21):3905-3916

80. Hirai M et al (2004) FOG-2 competes with GATA-4 for transcriptional coactivator p300 and represses hypertrophic responses in cardiac myocytes. J Biol Chem 279(36):37640-37650

81. Vakoc CR et al (2005) Proximity among distant regulatory elements at the beta-globin locus requires GATA-1 and FOG-1. Mol Cell 17(3):453-462

82. Tsang AP et al (1997) FOG, a multitype zinc finger protein, acts as a cofactor for transcription factor GATA-1 in erythroid and megakaryocytic differentiation. Cell 90(1):109-119

83. Crispino JD et al (1999) Use of altered specificity mutants to probe a specific protein-protein interaction in differentiation: the GATA-1:FOG complex. Mol Cell 3(2):219-228

84. Hong W et al (2005) FOG-1 recruits the NuRD repressor complex to mediate transcriptional repression by GATA-1. EMBO J 24(13):2367-2378

85. Yu M et al (2009) Insights into GATA-1-mediated gene activation versus repression via genome-wide chromatin occupancy analysis. Mol Cell 36(4):682-695

86. Sanalkumar R et al (2014) Mechanism governing a stem cellgenerating cis-regulatory element. Proc Natl Acad Sci USA 111(12):E1091-E1100

87. Lan X et al (2012) Integration of Hi-C and ChIP-seq data reveals distinct types of chromatin linkages. Nucleic Acids Res 40(16):7690-7704

88. Trivedi CM et al (2010) Hopx and Hdac2 interact to modulate Gata4 acetylation and embryonic cardiac myocyte proliferation. Dev Cell 19(3):450-459

89. Ozawa Y et al (2001) Histone deacetylase 3 associates with and represses the transcription factor GATA-2. Blood 98(7):2116-2123

90. Chen GY et al (2006) Interaction of GATA-3/T-bet transcription factors regulates expression of sialyl Lewis $\mathrm{X}$ homing receptors on Th1/Th2 lymphocytes. Proc Natl Acad Sci USA 103(45):16894-16899

91. Watamoto $\mathrm{K}$ et al (2003) Altered interaction of HDAC5 with GATA-1 during MEL cell differentiation. Oncogene 22(57):9176-9184

92. Dai YS, Markham BE (2001) p300 Functions as a coactivator of transcription factor GATA-4. J Biol Chem 276(40):37178-37185

93. Pasini D et al (2010) Characterization of an antagonistic switch between histone $\mathrm{H} 3$ lysine 27 methylation and acetylation in the transcriptional regulation of Polycomb group target genes. Nucleic Acids Res 38(15):4958-4969
94. Martowicz ML et al (2005) Dynamic GATA factor interplay at a multicomponent regulatory region of the GATA-2 locus. J Biol Chem 280(3):1724-1732

95. Tripic T et al (2009) SCL and associated proteins distinguish active from repressive GATA transcription factor complexes. Blood 113(10):2191-2201

96. Montgomery RL et al (2007) Histone deacetylases 1 and 2 redundantly regulate cardiac morphogenesis, growth, and contractility. Genes Dev 21(14):1790-1802

97. He A et al (2012) PRC2 directly methylates GATA4 and represses its transcriptional activity. Genes Dev 26(1):37-42

98. Nadeau $\mathrm{M}$ et al (2010) An endocardial pathway involving Tbx5, Gata4, and Nos3 required for atrial septum formation. Proc Natl Acad Sci USA 107(45):19356-19361

99. Sepulveda JL et al (1998) GATA-4 and Nkx-2.5 coactivate Nkx2 DNA binding targets: role for regulating early cardiac gene expression. Mol Cell Biol 18(6):3405-3415

100. Liu $\mathrm{F}$ et al (2015) GATA-binding factor 6 contributes to AV node development and function. Circ Cardiovasc Genet 8(2):284-293

101. Moskowitz IP et al (2011) Transcription factor genes Smad4 and Gata4 cooperatively regulate cardiac valve development (corrected). Proc Natl Acad Sci USA 108(10):4006-4011

102. Singh MK et al (2010) Gata4 and Gata5 cooperatively regulate cardiac myocyte proliferation in mice. $J$ Biol Chem 285(3): 1765-1772

103. Clabby ML et al (2003) Retinoid X receptor alpha represses GATA-4-mediated transcription via a retinoid-dependent interaction with the cardiac-enriched repressor FOG-2. J Biol Chem 278(8):5760-5767

104. Misra C et al (2014) Disruption of myocardial Gata4 and Tbx5 results in defects in cardiomyocyte proliferation and atrioventricular septation. Hum Mol Genet 23(19):5025-5035

105. Adamo RF et al (2004) GATA-6 gene enhancer contains nested regulatory modules for primary myocardium and the embedded nascent atrioventricular conduction system. Anat Rec A Discov Mol Cell Evol Biol 280(2):1062-1071

106. Singh $R$ et al (2009) Tbx20 interacts with smads to confine tbx2 expression to the atrioventricular canal. Circ Res 105(5):442-452

107. van Weerd JH et al (2014) A large permissive regulatory domain exclusively controls tbx 3 expression in the cardiac conduction system. Circ Res 115(4):432-441

108. Di Lisi R et al (2000) An atrioventricular canal domain defined by cardiac troponin I transgene expression in the embryonic myocardium. Anat Embryol (Berl) 202(2):95-101

109. Wang J, Sun YM, Yang YQ (2012) Mutation spectrum of the GATA4 gene in patients with idiopathic atrial fibrillation. Mol Biol Rep 39(8):8127-8135

110. Li J et al (2012) Novel GATA6 loss-of-function mutation responsible for familial atrial fibrillation. Int $\mathrm{J}$ Mol Med 30(4):783-790

111. Yang YQ et al (2012) GATA6 loss-of-function mutation in atrial fibrillation. Eur J Med Genet 55(10):520-526

112. Yang YQ et al (2012) Prevalence and spectrum of GATA6 mutations associated with familial atrial fibrillation. Int J Cardiol 155(3):494-496

113. McCulley DJ, Black BL (2012) Transcription factor pathways and congenital heart disease. Curr Top Dev Biol 100:253-277

114. Kostetskii I et al (1999) Retinoid signaling required for normal heart development regulates GATA-4 in a pathway distinct from cardiomyocyte differentiation. Dev Biol 206(2):206-218

115. Wang G, Yeh HI, Lin JJ (1994) Characterization of cis-regulating elements and trans-activating factors of the rat cardiac troponin T gene. J Biol Chem 269(48):30595-30603 
116. Fischer A, Gessler M (2007) Delta-Notch-and then? Protein interactions and proposed modes of repression by Hes and Hey bHLH factors. Nucleic Acids Res 35(14):4583-4596

117. Kokubo H et al (2007) Hesr1 and Hesr2 regulate atrioventricular boundary formation in the developing heart through the repression of Tbx2. Development 134(4):747-755

118. Rosen MR et al (2011) The road to biological pacing. Nat Rev Cardiol 8(11):656-666

119. Jung JJ et al (2014) Programming and isolation of highly pure physiologically and pharmacologically functional sinus-nodal bodies from pluripotent stem cells. Stem Cell Reports 2(5):592-605

120. Ieda $M$ et al (2010) Direct reprogramming of fibroblasts into functional cardiomyocytes by defined factors. Cell 142(3):375-386

121. Song $\mathrm{K}$ et al (2012) Heart repair by reprogramming non-myocytes with cardiac transcription factors. Nature 485(7400):599-604

122. Chen JX et al (2012) Inefficient reprogramming of fibroblasts into cardiomyocytes using Gata4, Mef2c, and Tbx5. Circ Res 111(1):50-55
123. Iwafuchi-Doi M, Zaret KS (2014) Pioneer transcription factors in cell reprogramming. Genes Dev 28(24):2679-2692

124. Cirillo LA et al (2002) Opening of compacted chromatin by early developmental transcription factors HNF3 (FoxA) and GATA-4. Mol Cell 9(2):279-289

125. Wang L et al (2015) Stoichiometry of Gata4, Mef2c, and Tbx5 influences the efficiency and quality of induced cardiac myocyte reprogramming. Circ Res 116(2):237-244

126. Huangfu D et al (2008) Induction of pluripotent stem cells by defined factors is greatly improved by small-molecule compounds. Nat Biotechnol 26(7):795-797

127. Zuryn S et al (2014) Transdifferentiation. Sequential histonemodifying activities determine the robustness of transdifferentiation. Science 345(6198):826-829

128. Buganim Y, Faddah DA, Jaenisch R (2013) Mechanisms and models of somatic cell reprogramming. Nat Rev Genet 14(6):427-439 